\title{
Narrative review of facial gender surgery: approaches and techniques for the frontal sinus and upper third of the face
}

\author{
Matthew Louis ${ }^{1}$, Roberto Travieso ${ }^{1,2}$, Norah Oles ${ }^{1,2}$, Devin Coon $^{1,2}$ \\ ${ }^{1}$ Department of Plastic and Reconstructive Surgery, Johns Hopkins University School of Medicine, Baltimore, MD, USA; ${ }^{2}$ Johns Hopkins Center for \\ Transgender Health, Johns Hopkins Hospital, Baltimore, MD, USA \\ Contributions: (I) Conception and design: M Louis, R Travieso, D Coon; (II) Administrative support: M Louis; (III) Provision of study materials or \\ patients: D Coon; (IV) Collection and assembly of data: M Louis, R Travieso, D Coon; (V) Data analysis and interpretation: M Louis, R Travieso, D \\ Coon; (VI) Manuscript writing: All authors; (VII) Final approval of manuscript: All authors. \\ Correspondence to: Devin Coon, MD, MSE. Department of Plastic Surgery, Johns Hopkins Hospital, 601 N. Caroline Street, JHOC 8161, Baltimore, \\ MD 21287, USA. Email: dcoon@jhmi.edu.
}

\begin{abstract}
Facial gender confirmation surgery (FGCS) is a series of procedures which seek to harmonize a patient's face with his/her self-image and gender identity. Originally described in San Francisco in the 1980s, FGCS has evolved to encompass all elements of the craniofacial skeleton and facial soft tissue. This field in plastic and reconstructive surgery has quickly gained more attention in the past decade due to the pioneering work of groups around the world along with increased social acceptance and medical care of the transgender community. This narrative review focuses on the upper third of the face. Key differences in the forehead and the hairline of cis men and women are discussed which inform pharmacologic and surgical interventions. Hairline modifying therapies including pharmacotherapy and hair transplantation are explained. Virtual surgical planning (VSP), a tool broadly used in surgical fields, has a special role in FGCS and we offer advice in using VSP when addressing the frontal sinus. Use of VSP allows the surgeon to provide reproducible and accurate results. We then discuss the history of the frontal sinus setback and offer our algorithmic approach to recontouring the forehead with detailed description of the operative steps and decision making. Finally, postoperative care and complications considered.
\end{abstract}

Keywords: Facial feminization; gender affirmation surgery; frontal sinus setback

Submitted Sep 15, 2020. Accepted for publication Feb 25, 2021.

doi: 10.21037/atm-20-6432

View this article at: http://dx.doi.org/10.21037/atm-20-6432

\section{Introduction}

\section{History of facial gender confirmation surgery (FGCS)}

What is now known as FGCS had its initial moniker as facial feminization surgery (FFS) and was a field pioneered by Dr. Douglas Ousterhout in San Francisco in the mid 1980s $(1,2)$. FFS was originally described as a series of procedures in order to feminize a transgender man's face which arose out of a need for validation of the transwomen in carrying out their new social role as a woman. In recent years, as transgender men have also sought facial harmonizing procedures, it became increasingly apparent that a more encompassing term was needed. Such was the advent of FGCS (3). As much has changed since the advent of FGCS, we seek to provide a concise review of FGCS in the modern context with special consideration to the medical necessity of FGCS, use of virtual surgical planning (VSP), and updated technical considerations.

Since these procedures have become more prevalent, there have been questions regarding the medical necessity of FGCS and the psychosocial outcomes these procedures generate. For many transgender patients the face is a source of significant dysphoria and addressing this can increase the patient's quality of life and unify his/her physical 


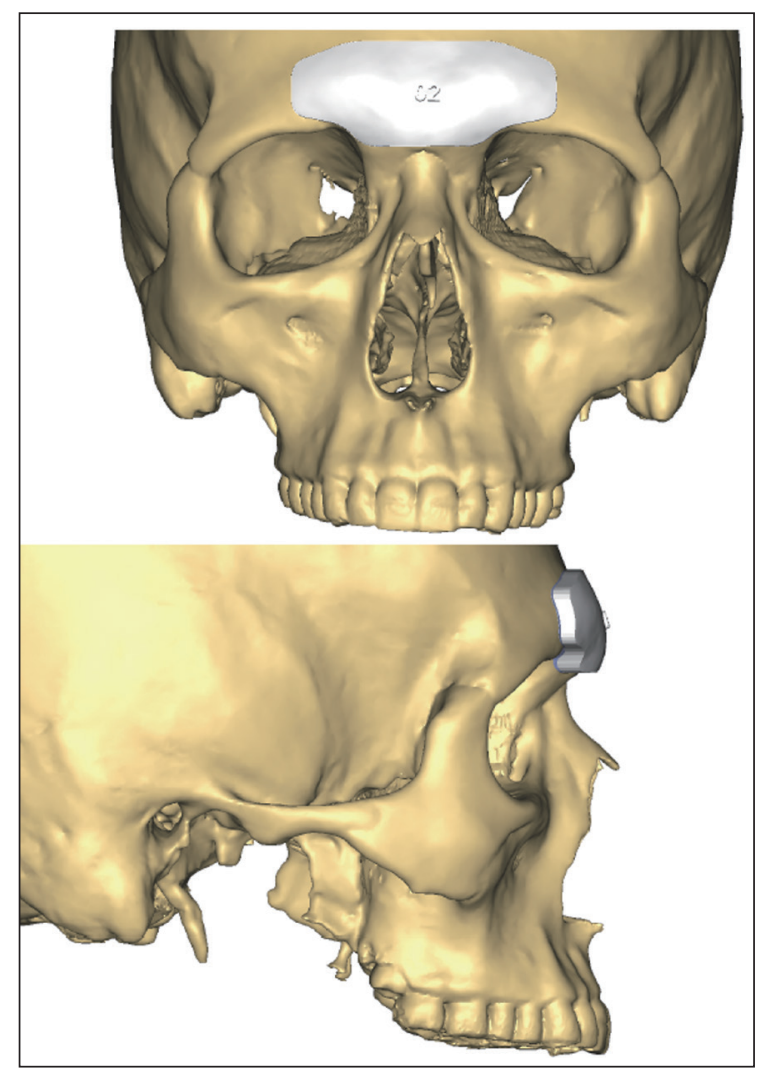

Figure 1 Frontal, lateral views of pre-operative virtual surgical planning for feminization of the forehead. The cutting guide, designed in VSP sessions, reduces the amount of time required to perform the osteotomy and decreases risk of intracranial violation.

features and self-image (4). Although there has only been one international, prospective, international, multicenter, cohort study on FFS demonstrating improved quality of life, the daily impact of the results of these procedures are unequivocally positive (5-12).

\section{Access to care and medical necessity}

As access to healthcare has increased for this population and patients are receiving treatment earlier, the need for FGCS has decreased secondary to the earlier use of hormone therapy (13). However, this has not obviated the need for more surgeons competent in performing FGCS or the need for expert consensus-even the current version of the WPATH standards of care (SOC7) does not explicitly deem FGCS as medically necessary. For these reasons, the first International Facial Gender Symposium (IFGS) was held in
2019, bringing together a multidisciplinary group including surgeons, psychologists, and researchers focused on the area of facial gender surgery. The expert consensus, based on literature review and aggregated experience spanning thousands of FGCS patients, explicitly states that FGCS procedures are medically necessary. It appeals for insurance coverage of these procedures for both facial feminization and masculinization with additional CPT codes, encourages interdisciplinary treatment teams, and calls for unambiguous standards defining adequate training to perform FGCS. It highlights the need for additional, high-quality research over all procedures encompassed by FGCS using validated tools and objective standards for outcomes evaluation.

Should FGCS be deemed medically necessary and insurance carriers begin covering these interventions, an increasing number of patients will be seeking this series of procedures. It is thus incumbent upon our specialty to ensure we provide the highest quality of care in treating these patients. FGCS is technically demanding and requires an amalgamation of skills ranging from craniofacial to aesthetic to general plastic surgery principles. FGCS thus has a steep learning curve $(2,3)$ and it is prudent to use any and all tools at one's disposal.

\section{Utility of VSP}

VSP is one such tool in the armamentarium with which many surgeons have familiarity. Using VSP and 3D analysis allows the surgeon to have a comprehensive understanding of the reconstructive problem well in advance of the day of operation (14). The surgeon can analyze the problem, plan the operation, print a model of the patient, practice osteotomies on the model, and develop cutting guides and custom plating systems. Figure 1 demonstrates one such cutting guide for frontal sinus setback. With all these advantages, VSP is a natural fit for FGCS as this series of operations requires precision and a detailed plan for achieving facial harmony.

We present the following article in accordance with the Narrative Review reporting checklist (available at http:// dx.doi.org/10.21037/atm-20-6432).

\section{Technical considerations}

The forehead is perhaps the anatomical area of the face that is most signifying of gender, and as such it was one of the first fields of FGCS to be pioneered and the techniques 
are very well described. Feminization of the forehead can be accomplished safely as long as one adheres to the fundamental principles.

\section{Anatomical differences in the forehead, scalp, and eyes}

The cis male and female forehead are quite different. The cis male forehead has varying degrees of supraorbital bossing transitioning cephalad to a concavity then convexity $(1-3,15)$. The cis female forehead generally lacks such topological differences and is more uniformly convex.

Hairlines can be distinctive markers of gender. Men most commonly have an $\mathrm{M}$-shaped hairline and varying degrees of male pattern baldness. Although women's hair thins and can undergo alopecia, the frontal hairline is generally oval in shape. While a varying degree of length of the upper third of the face is better tolerated in men, there is less tolerance with regards to a long forehead in women. Ideally, the hairline should be about $7-9 \mathrm{~cm}$ in the midline from glabella to hairline and about 5.5 to $5.8 \mathrm{~cm}$ above the brows in the midpoint $(16,17)$. The male forehead spans a larger distance, about $7-10 \mathrm{~cm}$ above the brows in the midpoint (18).

\section{Addressing the hairline, non-surgical options}

Hair loss is a distressing (and inevitable) event affecting all people, regardless of gender. It can be even more distressing to transwomen who have a discordant pattern of hair loss. Male patterned hair loss is defined by frontotemporal recession with eventual vertex alopecia $(19,20)$. Interestingly, androgen sensitivity and distribution of androgen receptors is region-specific in the scalp, and the occipital area is generally preserved (21). This is an important consideration that will be revisited when discussing hair transplantation.

Considering that $50 \%$ of patients beyond the age of 50 (regardless of assigned sex at birth) experience hair loss, it is likely that the transitioning woman will have some degree of "male" pattern balding and an M-shaped frontal hairline $(19,22)$. In general, when estrogen or hormone replacement therapy (HRT) is initiated, the balding progression is halted and patients may even experience marginal hair regrowth (23-26). Regardless of these factors, minoxidil, finasteride, and spironolactone may be useful adjuncts to prevent further regression and stimulate marginal reversal (27-31). Spironolactone, a commonly used androgen antagonist and diuretic, acts to decrease hair loss by blocking the activity of 5 -alpha-reductase thereby reducing the production of androgens and by directly blocking androgen receptors (32). Finasteride mitigates hair loss by blocking the action of 5 -alpha-reductase thereby diminishing the conversion of testosterone to dihydrotestosterone (DHT). Minoxidil, originally used as an anti-hypertensive, is an arteriolar vasodilator whose full mechanism of action is not yet clear (33). A 2016 Cochrane review found that of these medications, minoxidil has the highest quality of evidence regarding its efficacy; however, they noted that further studies were needed to assess quality of life improvements in each group as well as efficacy of finasteride and spironolactone (32). Moreover, there is a paucity of literature addressing minoxidil, finasteride, and spironolactone in the transgender patient.

\section{Addressing the bairline, surgical options}

The frontal hairline in a transwoman patient should be stable by the time of facial feminization, as hormone therapy will ideally have already been initiated for a year. There are three general options for surgically addressing the frontal hairline: follicular unit transplantation (FUT), follicular unit extraction (FUE), and hairline-lowering surgery (HLS). Each of these options can be safely done at time of frontal sinus setback or can be done in a separate procedure. Although some colleagues prefer to use FUT at time of frontal sinus setback and report good outcomes (34), other groups prefer to wait at least 6 months after frontal sinus setback. Of note, FUT and FUE are time-consuming in themselves and will add multiple hours to an already lengthy surgery. We have had patients undergo FUT on post-operative day one with another surgeon using the strip harvested by us the prior day and cryopreserved; however, engraftment success is likely somewhat diminished due to the longer duration between extraction and engraftment. FUT involves the resection of a portion of the scalp about $10-15 \mathrm{~mm}$ wide, sectioning the strip, and extracting each follicular unit (35). This necessitates access to a dedicated team that prepares each minigraft (3-4 hairs) or micrograft (1-2 hairs). While the grafts are back-tabled, the surgeon can prepare the recipient site in the desired area by making a hole with a 19-gauge needle. The depth should be $4 \mathrm{~mm}$ and the trajectory are site dependent (20-40 degrees from scalp, angled down in the frontal aspect, angled down in lateral aspects.) Less relevant to the transfeminine patient but still a worthy consideration when engrafting is the fact that native hair in the frontal hair will continue to thin while hair harvested from the occipital and parietal scalp will not, 
Table 1 Ousterhout description of the four types of foreheads and approaches to feminization of these foreheads

\begin{tabular}{ll}
\hline Type description & Management \\
\hline Type I: absent frontal sinusor sufficient bone over sinus (3-5\%) & Burring \\
Type II: Brow bossing at appropriate level, insufficientprojection superior to bossing (3-5\%) & Fill with methylmethacrylate vs. hydroxyapatite \\
Type III: Prominent bossing, presence of frontal sinus (90\%) & Frontal sinus setback \\
Type IV: Forehead small, under projected brow ridge (rare) & Pan forehead augmentation \\
\hline
\end{tabular}

as the engrafted hair maintains its donor site characteristics. If further frontal hair loss is not mitigated, the engrafted hairs will stand alone, sparse, and quite obvious as the native hairline recedes.

FUE is similar in engraftment principles but is different in that each follicular unit is individually harvested using a small coring device. This can be done sharply or bluntly, and each option has a manual and power assisted option. The sharp technique leads to a greater likelihood of follicle transection while the blunt technique leads to a higher rate of graft burial (7\%) (36). FUE is preferred in patients wishing to mitigate the appearance of a fine scar and wants to wear his/her hair short; this is likely a moot point in the patient who has had forehead reshaping.

HLS is a procedure that was originally developed to lower genetically high hairlines in women (17). This procedure can advance the scalp about $22 \mathrm{~mm}$ when combined with intraoperative creep, galeotomies, and securing the galea to the cranium with clips. When done in the cis-gender population with oval shaped frontal hairlines, patients are generally quite pleased; however, up to $50 \%$ still have FUE to fill in around the temporal areas and thicken the frontal hairline (17). Hairline lowering surgery is typically done in conjunction with frontal bone reduction but can be done independently if the transfemale patient does not desire frontal bone reduction and has an oval shaped hairline.

Each method has its place when considering the transgender patient and incision selection varies by center, surgeon preference, and availability of ancillary support (i.e., staff for FUT, FUE). If the patient has an $M$ shaped hairline, FUT at time of frontal bone reshaping is less favorable as closure of the $M$ sides limits the amount of lowering that can be achieved. If this is not feasible, FUT or FUE or FUT and FUE can be done once the incision is healed. Hairline lower surgery is ideal when the patient has an oval shaped hairline and does not wear their hair back where the scar can be visible.
If the hairline is not going to be specifically addressed at time of forehead feminization, we prefer and recommend a coronal incision. If the hairline is to be addressed with hairline lowering surgery, a pretrichial incision will be necessary.

\section{Forehead anatomy}

In his seminal 1987 paper, Ousterhout introduced a four part categorization system for transfeminine foreheads which dictates surgical management (1). This system can be appreciated in Table 1. However, as the field has evolved, fewer and fewer high-volume centers are utilizing PMMA and pan-forehead augmentation; most centers are using a frontal sinus setback except in cases of frontal sinus agenesis.

\section{Role of VSP}

VSP is quite useful in approaching the forehead as the presence and location of the frontal sinus can be considered pre-operatively. Custom jigs can be designed with the engineers in the VSP sessions and used in the operating room to make quick work of the osteotomies (Figure 1). The risk of intracranial violation during burring and osteotomies can be nearly obviated as the thickness and location of the posterior table and the frontal bone are already known. In one experiment using VSP for FGCS in a cadaver model, the frontal sinus setback in the study arm using intraoperative cutting guides took $19 v s$. 44 minutes in the group without the guides; moreover, there was no intracranial entry in the VSP group $v s$. a $12 \%$ incidence in the group without VSP (15).

\section{Forebead reshaping with frontal sinus setback and selective burring}

The hairline and planning one's incision warrant discussion as scalp advancement and forehead skin resection with 


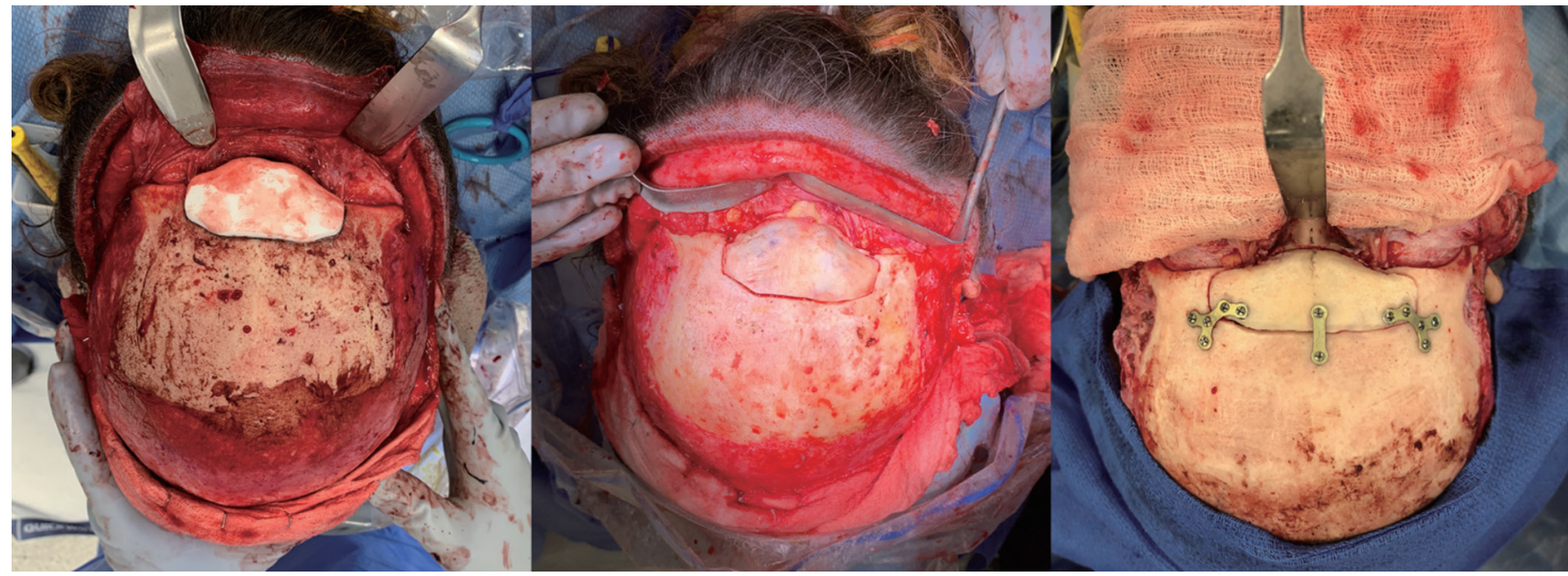

Figure 2 Intraoperative views. Left: pericranial flap is raised and cutting guide is situated over the frontal sinus. Middle: bone flap over frontal sinus after osteotomy. Right: bone flap after burring and fixation with miniplates in a more inferior, posteriorly rotated position.

a trichophytic incision will decrease the length of the forehead, while a coronal incision may lengthen the forehead; there is still debate in the literature regarding which is to be preferred (37-39). As discussed previously, we generally prefer the coronal approach espoused by Capitán; however, we will use a pretrichial approach as indicated and if requested by the patient.

Before incising the scalp, standard tumescent fluid (containing epinephrine and lidocaine) is instilled under the scalp to enhance hemostasis and facilitate hydrodissection. In planning the incision, a strip of scalp can be resected if desired for FUT or as needed for scalp advancement. A trichophytic incision should be used (beveling the knife 45 degrees at the skin.) A subgaleal plane is then entered and this is carried forward to the nasofrontal junction in the midline, and just under the superior orbital rims laterally to facilitate burring and possible ostectomy of the superior lateral orbital rim. A rectangular pericranial flap is then marked out encompassing the frontal bone and extending laterally and anteriorly to the fronto-zygomatic suture. This flap can generally be raised sharply.

Next, the supraorbital nerve must be identified and protected. If there is a true supraorbital foramen, osteotomies around the foramen will be required to free up the nerve and bring it anteriorly with the scalp flap. In some cases, it is better to transect and repair the nerve if it would require extensive osteotomy to mobilize from a very superiorly located foramen. Once adequate exposure is obtained, the osteotomy for the anterior table of the frontal sinus should be marked out. The osteotomies are accomplished using a piezoelectric saw (Synthes, Satelec, West Chester, PA, USA) If the bone flap does not come off freely, an osteotome may be used to complete the osteotomies and free up any attachments of the bone flap to the sinus or septum.

The anterior surface of the bone flap may then be burred to reduce its thickness. One should be judicious in this burring as an overly-thin bone flap is more prone to resorption and problems in the future. The trabecular network in the sinus should be reduced to allow for adequate inferior and posterior rotation of the bone flap once fixated. The surrounding frontal bone may then be burred to a uniform thickness. Comfort with this step is facilitated by practice and a systematic approach. We prefer to draw a grid on the frontal bone to help guide the amount of burring done. Without this systematic approach, differential depths of burring are more common. The nasofrontal angle can be reduced with burring at this point. Figure 2 demonstrates the overlaying of the cutting guide, the osteotomy as planned, and rigid fixation.

Attention should then be paid to the superior lateral orbital rim. Although the male orbital volume is generally larger, women have a proportionally larger orbital opening compared to the rest of the face. As such, an ostectomy or burring of the superior lateral orbital rim can be done. Once satisfied with the burring, one should rigidly fixate 
the anterior wall of the frontal sinus in its new inferior and posteriorly rotated position. We use four-hole miniplates and miniscrews. Mesh is used in areas where there is concern for thin bone that may resorb. Other prefer wire fixation; however, miniplates are well tolerated in this area and provide rigid fixation, reducing the likelihood of resorption or malposition. We deprecate the use of suture fixation which is non-rigid and poses greater risks of resorption.

The scalp should then be redraped and secured to the skull to set the brow position and arch. We prefer to use Endotines (MicroAire Surgical Instruments, Charlottesville, VA, USA) These are placed in the hair bearing portion of the temporal scalp and will resorb. They are well tolerated. Attention should be paid in this step to ensure that the surgeon is satisfied with the brow position. Cortical tunnels with resorbable suture represent an acceptable alternative. The wound is then closed over a single closed suction drain.

\section{Postoperative care}

The patient is kept for observation overnight and the drain is removed on postoperative day one. Head of bed elevation is imperative to reduce swelling. Regardless, swelling can be quite significant but is amenable to halotherapy. The patient receives perioperative antibiotics but will not be sent home from the hospital on antibiotics unless the patient has had a concurrent rhinoplasty in which internal nasal splints are utilized.

As previously mentioned, we prefer a frontal sinus setback whenever there is a frontal sinus present. Burring alone is not enough to feminize the forehead or else risks anterior table resorption from excessive thinning. The power of frontal sinus setback lays in the ability to lever the anterior wall of the frontal sinus posteriorly which creates significant changes in the appearance of the brow. Figure 3 demonstrates typical results of frontal sinus setback to achieve facial feminization.

\section{Complications}

Feminization of the forehead is a safe and effective procedure but can be associated with complications. Although reports of complications in the literature are rare, forehead feminization is not without its perils: regional anesthesia (supraorbital nerves), cerebrospinal fluid rhinorrhea, nonunion and malunion, infection (when using alloplastic materials), and alopecia are the most common $(9,40)$. Nonunion and mobility of the anterior table of the frontal sinus is one of the most common complications but is amenable to revision (41). Infection is more commonly encountered when using alloplastic materials to treat a Type IV forehead as discussed above. In Altman's discussion of his experience with forehead feminization, he had a $5 \%$ rate of cerebrospinal fluid rhinorrhea (40). If the rhinorrhea does not stop spontaneously, reoperation is necessary. If a dural rent is encountered during the frontal sinus setback, it can be repaired with sutures and a fibrin glue along with Surgicel (Ethicon) (40). However, there are also a number of case series of between 29 and 172 procedures in which no complications were encountered $(1,9,38)$.

\section{Outcomes}

In a forthcoming review and evidenced-based consensus statement with other high-volume centers, we found that satisfaction with FGCS was high (97\%, 534/550 patients, 11 studies, article in press). In a case-control study by Ainsworth and Spiegel from 2010, self-image and selfassessment of social aspects of facial appearance scores of patients who had underwent FGCS were compared with those who had not (5). Patients who had undergone these procedures had a clinically and statistically significant favorable difference from the control group.

In the first study to assess prospective outcomes of FGCS, Morrison et al. evaluated facial feminization outcome scores, photogrammetric cephalometrics, selfperceived masculinity and femininity, externally rated gender appearance, and general aesthetics of 66 consecutive patients in an international, multicentered trial (12). All outcomes were favorable in the six months follow-up period with median facial feminization outcome scores increasing from 47.2 to 80.6 (scale out of 100 , higher scores denote more feminine appearance.)

\section{Conclusions}

Craniofacial contouring of the forehead is a powerful surgical tool in harmonizing patients' facial aesthetics to their gender. Since the forehead and hairline are some of the most notable social markers for gender, reducing the supraorbital bossing common in the cis male and changing a M-shaped hairline to an oval one can significantly transform the upper third. VSP is a useful adjunct to help 


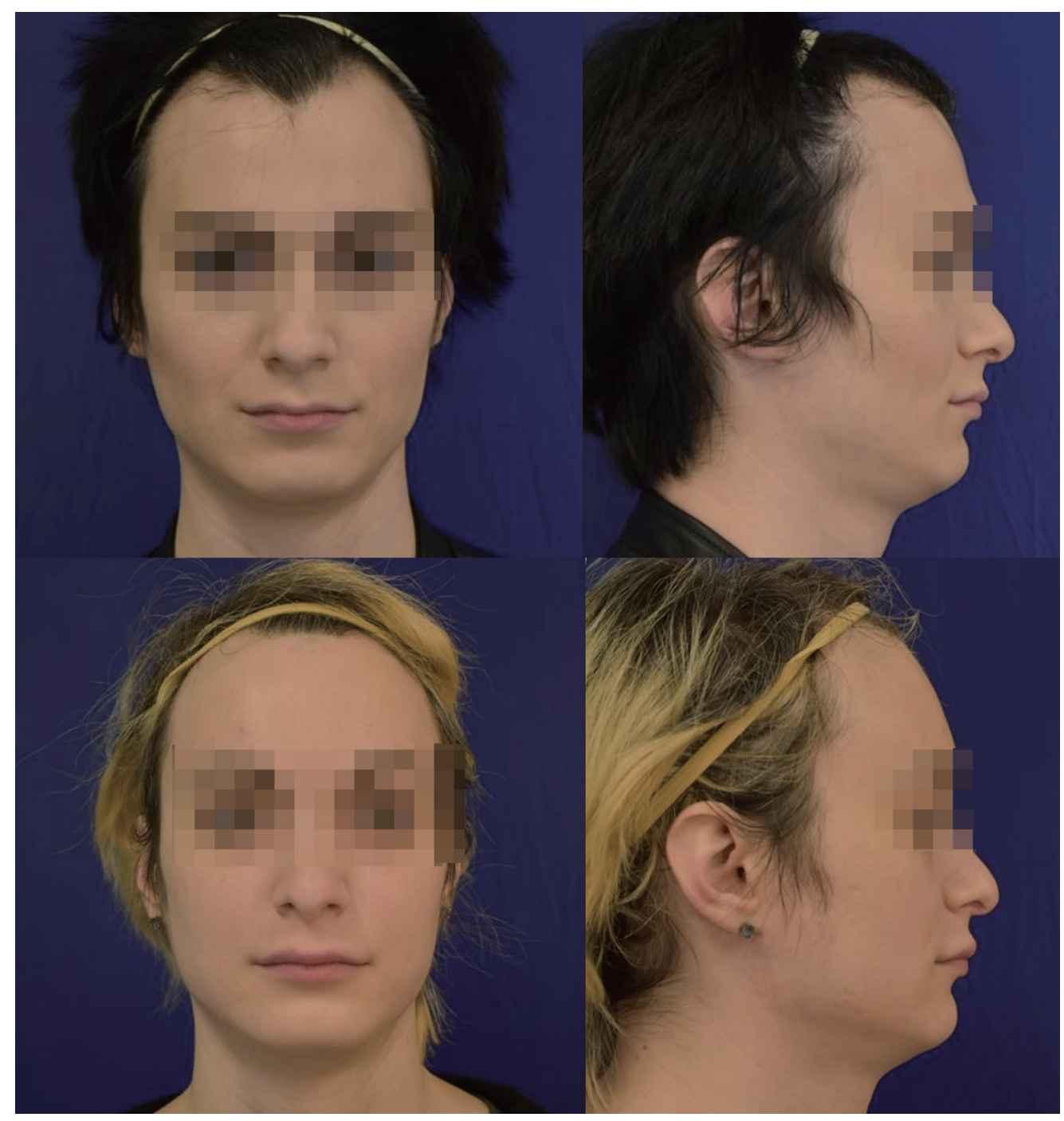

Figure 3 Top panel: pre-operative photos of a patient who underwent forehead feminization with a frontal sinus setback and burring of the frontal bone. Bottom panel: one-month post-operative photos demonstrating reduced frontal bossing and creation of a feminine, convex forehead.

surgeons more rapidly gain familiarity with the anatomy and osteotomies.

\section{Acknowledgments}

Funding: None.

\section{Footnote}

Provenance and Peer Review: This article was commissioned by the Guest Editors (Drs. Oscar J. Manrique, John A
Persing, and Xiaona Lu) for the series "Transgender Surgery" published in Annals of Translational Medicine. The article has undergone external peer review.

Reporting Checklist: The authors have completed the Narrative Review reporting checklist. Available at http:// dx.doi.org/10.21037/atm-20-6432

Conflicts of Interest: All authors have completed the ICMJE uniform disclosure form (available at http://dx.doi. org/10.21037/atm-20-6432). The series "Transgender 
Surgery" was commissioned by the editorial office without any funding or sponsorship. Dr. Louis reports grants from DePuy Synthes Trauma/Craniomaxillofacial Research Fellowship Program, during the conduct of the study. The authors have no other conflicts of interest to declare.

Ethical Statement: The authors are accountable for all aspects of the work in ensuring that questions related to the accuracy or integrity of any part of the work are appropriately investigated and resolved.

Open Access Statement: This is an Open Access article distributed in accordance with the Creative Commons Attribution-NonCommercial-NoDerivs 4.0 International License (CC BY-NC-ND 4.0), which permits the noncommercial replication and distribution of the article with the strict proviso that no changes or edits are made and the original work is properly cited (including links to both the formal publication through the relevant DOI and the license). See: https://creativecommons.org/licenses/by-nc-nd/4.0/.

\section{References}

1. Ousterhout DK. Feminization of the forehead: contour changing to improve female aesthetics. Plast Reconstr Surg 1987;79:701-13.

2. Deschamps-Braly J. Facial Gender Affirmation Surgery: Facial Feminization Surgery and Facial Masculinization Surgery. In: Schechter LS, editor. Gender Confirmation Surgery: Principles and Techniques for an Emerging Field [Internet]. Cham: Springer International Publishing; 2020 [cited 2020 Mar 31]. p. 99-113. Available online: https:// doi.org/10.1007/978-3-030-29093-1_12

3. Deschamps-Braly JC. Approach to Feminization Surgery and Facial Masculinization Surgery: Aesthetic Goals and Principles of Management. J Craniofac Surg 2019;30:1352-8.

4. Padula WV, Heru S, Campbell JD. Societal Implications of Health Insurance Coverage for Medically Necessary Services in the U.S. Transgender Population: A CostEffectiveness Analysis. J Gen Intern Med 2016;31:394-401.

5. Ainsworth TA, Spiegel JH. Quality of life of individuals with and without facial feminization surgery or gender reassignment surgery. Qual Life Res 2010;19:1019-24.

6. Berli JU, Plemons E. The Importance of Facial Gender Confirmation Surgery. In: Schechter LS, editor. Gender Confirmation Surgery: Principles and Techniques for an Emerging Field [Internet]. Cham: Springer International
Publishing; 2020 [cited 2020 Mar 31]. p. 91-7. Available online: https://doi.org/10.1007/978-3-030-29093-1_11

7. Berli JU, Capitán L, Simon D, et al. Facial gender confirmation surgery-review of the literature and recommendations for Version 8 of the WPATH Standards of Care. Int J Transgend 2017;18:264-70.

8. Raffaini M, Magri AS, Agostini T. Full Facial Feminization Surgery: Patient Satisfaction Assessment Based on 180 Procedures Involving 33 Consecutive Patients. Plast Reconstr Surg 2016;137:438-48.

9. Morrison SD, Vyas KS, Motakef S, et al. Facial Feminization: Systematic Review of the Literature. Plast Reconstr Surg 2016;137:1759-70.

10. Isung J, Möllermark C, Farnebo F, et al. Craniofacial Reconstructive Surgery Improves Appearance Congruence in Male-to-Female Transsexual Patients. Arch Sex Behav 2017;46:1573-6.

11. Morrison SD, Crowe CS, Wilson SC. Consistent Quality of Life Outcome Measures Are Needed for Facial Feminization Surgery. J Craniofac Surg 2017;28:851-2.

12. Morrison SD, Capitán-Cañadas F, Sánchez-García A, et al. Prospective Quality-of-Life Outcomes after Facial Feminization Surgery: An International Multicenter Study. Plast Reconstr Surg 2020;145:1499-509.

13. Rosenthal SM. Approach to the patient: transgender youth: endocrine considerations. J Clin Endocrinol Metab 2014;99:4379-89.

14. Steinbacher DM. Three-Dimensional Analysis and Surgical Planning in Craniomaxillofacial Surgery. J Oral Maxillofac Surg 2015;73:S40-56.

15. Gray R, Nguyen K, Lee JC, et al. Osseous Transformation with Facial Feminization Surgery: Improved Anatomical Accuracy with Virtual Planning. Plast Reconstr Surg 2019;144:1159-68.

16. Nusbaum BP, Fuentefria S. Naturally occurring female hairline patterns. Dermatol Surg 2009;35:907-13.

17. Epstein J, Epstein GK. Hairline-Lowering Surgery. Facial Plast Surg Clin North Am 2020;28:197-203.

18. Unger WP. Hair Transplantation: Current Concepts and Techniques. J Investig Dermatol Symp Proc 2005;10:225-9.

19. Norwood OT. Male pattern baldness: classification and incidence. South Med J 1975;68:1359-65.

20. Gupta M, Mysore V. Classifications of Patterned Hair Loss: A Review. J Cutan Aesthet Surg 2016;9:3-12.

21. Sawaya ME, Price VH. Different levels of 5alphareductase type I and II, aromatase, and androgen receptor in hair follicles of women and men with androgenetic 
alopecia. J Invest Dermatol 1997;109:296-300.

22. Norwood OT. Incidence of Female Androgenetic Alopecia (Female Pattern Alopecia). Dermatol Surg 2001;27:53-4.

23. Lynfield YL. Effect of pregnancy on the human hair cycle. J Invest Dermatol 1960;35:323-7.

24. Niiyama S, Happle R, Hoffmann R. Influence of estrogens on the androgen metabolism in different subunits of human hair follicles. Eur J Dermatol 2001;11:195-8.

25. Giltay EJ, Gooren LJ. Effects of sex steroid deprivation/ administration on hair growth and skin sebum production in transsexual males and females. J Clin Endocrinol Metab 2000;85:2913-21.

26. Gao Y, Maurer T, Mirmirani P. Understanding and Addressing Hair Disorders in Transgender Individuals. Am J Clin Dermatol 2018;19:517-27.

27. Kanti V, Hillmann K, Kottner J, et al. Effect of minoxidil topical foam on frontotemporal and vertex androgenetic alopecia in men: a 104-week open-label clinical trial. J Eur Acad Dermatol Venereol 2016;30:1183-9.

28. Katz HI, Hien NT, Prawer SE, et al. Long-term efficacy of topical minoxidil in male pattern baldness. J Am Acad Dermatol 1987;16:711-8.

29. Stevenson MO, Wixon N, Safer JD. Scalp Hair Regrowth in Hormone-Treated Transgender Woman. Transgend Health 2016;1:202-4.

30. Adenuga P, Summers P, Bergfeld W. Hair regrowth in a male patient with extensive androgenetic alopecia on estrogen therapy. J Am Acad Dermatol 2012;67:e121-3.

31. Price VH. Treatment of hair loss. N Engl J Med 1999;341:964-73.

Cite this article as: Louis M, Travieso R, Oles N, Coon D. Narrative review of facial gender surgery: approaches and techniques for the frontal sinus and upper third of the face. Ann Transl Med 2021;9(7):606. doi: 10.21037/atm-20-6432
32. van Zuuren EJ, Fedorowicz Z, Schoones J. Interventions for female pattern hair loss. Cochrane Database Syst Rev [Internet]. 2016 May 26 [cited 2020 Sep 13];2016(5). Available online: https://www.ncbi.nlm.nih.gov/pmc/ articles/PMC6457957/

33. Suchonwanit $P$, Thammarucha S, Leerunyakul K. Minoxidil and its use in hair disorders: a review. Drug Des Devel Ther 2019;13:2777-86.

34. Capitán L, Simon D, Meyer T, et al. Facial Feminization Surgery: Simultaneous Hair Transplant during Forehead Reconstruction. Plast Reconstr Surg 2017;139:573-84.

35. Sand JP. Follicular Unit Transplantation. Facial Plast Surg Clin North Am 2020;28:161-7.

36. Harris JA. Follicular unit extraction. Facial Plast Surg Clin North Am 2013;21:375-84.

37. Ousterhout DK. Facial Feminization Surgery: The Forehead. Surgical Techniques and Analysis of Results. Plast Reconstr Surg 2015;136:560e-1e.

38. Capitán L, Simon D, Kaye K, et al. Facial feminization surgery: the forehead. Surgical techniques and analysis of results. Plast Reconstr Surg 2014;134:609-19.

39. Capitán L, Simon D, Kaye K, et al. Reply: Facial Feminization Surgery. Plast Reconstr Surg 2015;136:561e-3e.

40. Altman K. Forehead reduction and orbital contouring in facial feminisation surgery for transgender females. Br J Oral Maxillofac Surg 2018;56:192-7.

41. Spiegel JH. Facial determinants of female gender and feminizing forehead cranioplasty. Laryngoscope 2011;121:250-61. 